\title{
Quantum correlations of two qubits interacting with a macroscopic medium
}

\author{
Yan Liu, ${ }^{1}$ Jing Lu, ${ }^{1, *}$ and Lan Zhou ${ }^{1}$ \\ ${ }^{1}$ Key Laboratory of Low-Dimensional Quantum Structures and Quantum Control of Ministry of Education, \\ and Department of Physics, Hunan Normal University, Changsha 410081, China
}

(Dated: June 17, 2021)

\begin{abstract}
We consider two particles of spin-1/2 interacting with a one-dimensional $N$-spin array, which is an exactly solvable model. The dynamics of entanglement and quantum discord (QD) of the spins of the two particles is investigated by regarding the $1 \mathrm{D} \mathrm{N}$-spin array as the environment. It is found that although the entanglement may suffers a sudden death and a sudden birth in the evolution, it can neither be generated nor become larger than its initial value. Different from the entanglement dynamics, QD can be amplified, and even be generated by the interaction between particles and the common environment. We also observe that QD decays asymptotically to zero and later experiences a rival when the average number of excitation in the $1 \mathrm{D} \mathrm{N}$-spin array becomes larger in the case of nonzero inter-distance between two particles.

PACS numbers: 03.67.-a, 03.65.Ta, 03.65.Yz
\end{abstract}

\section{INTRODUCTION}

To find the fundamental resource for quantum information processing tasks, quantum entanglement has been regarded as a necessary prerequisite in order for quantum correlations to occur. With the doubting on entanglement being responsible for all quantum speedups raised by Knill and Laflamme [1], it is discovered that superposition principle can entail an alternative type of nonclassical correlation apart from entanglement. There are several measure to characterize all nonclassical correlations [2]. Quantum discord [3, 4] is the prominent one, which is defined as the difference between the quantum generalizations of two classically equivalent versions of the mutual information. Quantum discord is original introduced to be an information-theoretic approach to decoherence mechanisms in a quantum measurement process.

A quantum measurement process typically involves a measured system and a measuring apparatus, where the measured system is described as a quantum system and the apparatus behaves as a classical object. And the process of measurement can be described as a dynamic evolution process via an appropriate coupling between a measured system and the measuring apparatus. To draw light on the border between the quantum and the classical, the macroscopic character of the apparatus is taken into account by Hepp and Coleman. In their exactly solvable model, known as the Coleman-Hepp $(\mathrm{CH})$ model [5], the apparatus is a "large system" and the number of its constituting components approaches infinity, where each component obeys the Schrödinger equation and interactes with the measured system locally. Later on, a modified version of $\mathrm{CH}$ model is proposed by Nakazato and Pascazio [6], which takes energy exchange between the measured system and the apparatus into account. Based on exact solvability of the ColemanHepp model and its generalized version [6-8], the emergence of classicality of a quantum system is due to the elimination of the off-diagonal elements of the density matrix, which is characterized by a factorization structure [7] due to the inter-

${ }^{*}$ Corresponding author; Electronic address: lujing@hunnu.edu.cn action between the quantum system and the classical object. And this factorization gives rise to the exponential behavior of a quantum system [9]. However, to take the information transfer associated with the measured system and the apparatus into account, a triple model is necessary for a quantum measurement process, which consists of a measured system, an apparatus, and an environment. This invokes the investigation of the behavior of correlations of a two-qubit composite system exposed to noisy environments [10-20]. However, many of these studies concern on independent environments, and approximations are usually employed.

Exactly solvable models, which exclude the effects of approximations, not only gives us good insights into physics, but they also provide us with a better understanding of the complicated phenomena involved, for example, the $\mathrm{CH}$ model and its generalized version [6-8] have given us a comprehensive understanding of the quantum measurement processes. In this paper, we study two particles with spin-1/2 interacting with a one-dimensional (1D) $\mathrm{N}$-spin array, which is a generalized version of $\mathrm{CH}$ model. We regarded the spins of two particles and the 1D $N$-spin array as a two-qubit composite system and its surrounding environment, respectively. The 1D $N$-spin array is a macroscopic system as $N$ becomes larger. To introduce the macroscopic medium of the 1D array, we first study the effect of the macroscopic system on one qubit, it is found that the dynamic evolution of the qubit can be modeled as a phase damping channel in the weak-coupling macroscopic limit. Next, quantum correlations of the spins of two particles are investigated. It is found that quantum discord can be amplified, even generated via the interaction. We also observed the revival of quantum discord and entanglement under some conditions.

This paper is organized as follows. In Sec.II we introduce the model-two particles with spin-1/2 interacting with $1 \mathrm{D}$ array made of $N$ identical spins. In Sec. III we derive the time evolution operator of the total system, and also present the damping effect of the 1D array on the spin state of one particle. In Sec. IV] we study quantum correlation of the twoqubit in the time evolution. Finally, We conclude this work in Sec. V. 


\section{THE MODEL}

The $\mathrm{CH}$ model describes an ultrarelativistic particle interacting with $1 \mathrm{D}$ array of $N$ identical spins. Each spin of the 1D array could be regarded as a $\mathrm{AgBr}$ molecule with the down (up) state corresponding to the undivided (dissociated) molecule [5]. In the present generalized version, we consider two particles $Q_{A}$ and $Q_{B}$. Each particle possesses a spin, and moves in the $x$ direction with a positive constant velocity $v_{j}$ $(j=A, B)$. The 1D $N$-spin array has the finite size with length $L=x_{N}-x_{1}$, where $x_{1}\left(x_{N}\right)$ is the position of the first (final) spin of the array. After the particles arriving at position $x_{1}>0$, the particles begin their interaction with the spin array.

The Hamiltonian of the total system

$$
H=H_{Q}+H_{E}+H^{\prime}
$$

is a sum of three parts, where

$$
H_{Q}=v_{A} \hat{p}_{A}+v_{B} \hat{p}_{B}+\sum_{j=A, B} \frac{1}{2} \hbar \omega_{j}\left(1+\tau_{j}^{z}\right)
$$

is the free Hamiltonian of two particles,

$$
H_{E}=\frac{1}{2} \hbar \omega \sum_{n=1}^{N}\left(1+\sigma_{n}^{z}\right)
$$

is the free Hamiltonian of 1D N-spin array, and

$$
\begin{aligned}
H^{\prime} & =\sum_{j=A, B} \frac{1+\tau_{j}^{z}}{2} H_{j E}^{\prime} \\
& \equiv \sum_{j=A, B} \sum_{n=1}^{N} \frac{1+\tau_{j}^{z}}{2} V\left(\hat{x}_{j}-x_{n}\right)\left(\sigma_{n}^{+} e^{-i \frac{\omega}{v_{j}} \hat{x}_{j}}+\text { h.c. }\right)
\end{aligned}
$$

is the interaction Hamiltonian between the particles and the 1D $N$-spin array. Here, $\sigma_{n}^{\alpha}(\alpha= \pm, z)$ is the Pauli spin operators for the $n$th spin of the 1D array, $\tau_{j}^{z}$ is the Pauli matrice acting on the spin of the $j$ th particle. $\hat{x}_{j}$ and $\hat{p}_{j}$ are the coordinator and momentum operator of the $Q_{j}$ particle, which satisfies the canonical commutation relation $\left[\hat{x}_{j}, \hat{p}_{j^{\prime}}\right]=i \hbar \delta_{j j^{\prime}}$. The real function $V\left(\hat{x}_{j}-x_{n}\right)$ characterizes the strength of the interaction between the particles and each spin of the 1D array, where $x_{n}(n=1, \ldots, N)$ are the positions of the spin in the array $\left(x_{n}>x_{n-1}\right)$. Hereafter, the subscript $n$ denotes the spatial location of each spin in the array.

Comparing to the original $\mathrm{CH}$ model, the interaction Hamiltonian is changed to taking the spins of the particles into account, which describes that the $j$ th particle at the position $x$ exerts a real potential $V\left(\hat{x}_{j}-x_{n}\right)$ on the $n$th spin if and only if particle's spin is up [8]. Denoting $\left|p_{j}\right\rangle$ as the state that the $j$ th particle has momentum $p$ and $\left|0_{n}\right\rangle\left(\left|1_{n}\right\rangle\right)$ as the down (up) state of the $n$th spin, Hamiltonian $H_{j n}^{\prime}$ changes the states as

$$
\begin{aligned}
& H_{j n}^{\prime}\left|p_{j}\right\rangle\left|0_{n}\right\rangle=V\left(\hat{x}_{j}-x_{n}\right)\left|p_{j}-\frac{\hbar \omega}{v_{j}}\right\rangle\left|1_{n}\right\rangle, \\
& H_{j n}^{\prime}\left|p_{j}\right\rangle\left|1_{n}\right\rangle=V\left(\hat{x}_{j}-x_{n}\right)\left|p_{j}+\frac{\hbar \omega}{v_{j}}\right\rangle\left|0_{n}\right\rangle,
\end{aligned}
$$

where Hamiltonian

$$
H_{j n}^{\prime}=V\left(\hat{x}_{j}-x_{n}\right)\left(\sigma_{n}^{+} e^{-i \frac{\omega}{v_{j}} \hat{x}_{j}}+\text { h.c. }\right)
$$

is the $H_{j E}^{\prime}$ term acting on the $n$th spin and the $j$ th particle. Consequently, once the particles' spin is up, the interaction Hamiltonian $H_{j E}^{\prime}$ given by Eq. (4) induces the energy exchange between the particles and the spin array due to the nonvanishing energy gap $\hbar \omega$ between the two states of the molecule [6]. The particle's spin undergoes free precession with frequency $\omega_{j}$.

For later convenience, the Hamiltonian of the total system is rearranged as the sum of the free Hamiltonian $H_{0}$ and the interaction Hamiltonian $H^{\prime}$ of the particle-array system

$$
H=H_{0}+H^{\prime},
$$

where $H_{0}=H_{Q}+H_{E}$.

\section{THE DYNAMICS OF THE TOTAL SYSTEM}

For a given initial state of the total system $\rho(0)$, the state at time $t$ reads $\rho(t)=U(t) \rho(0) U^{\dagger}(t)$. Obviously, the evolution operator $U(t)=e^{-i H t / \hbar}$ characterizes the dynamical of the particle-array system, where the initial time is set as $t_{0}=0$. To exactly solve the present model, we introduce the interaction picture by writing the evolution operator as $U(t)=e^{-i H_{0} t / \hbar} U_{A B}$, where the unitary operator $U_{A B}(t)$ satisfies the Schrödinger equation with the Hamiltonian (4). Since the total spin of the particles $Q_{A}$ and $Q_{B}$ along the $z$ direction is always conserved during the time evolution, we decompose the unitary operator as

$$
\begin{aligned}
U_{A B}(t)= & U_{A}(t) U_{B}(t)|\uparrow \uparrow\rangle\langle\uparrow \uparrow|+| \downarrow \downarrow\rangle\langle\downarrow \downarrow|+ \\
& U_{B}(t)|\downarrow \uparrow\rangle\left\langle\downarrow \uparrow\left|+U_{A}(t)\right| \uparrow \downarrow\right\rangle\langle\uparrow \downarrow|,
\end{aligned}
$$

where states $|\downarrow\rangle$ and $|\uparrow\rangle$ are the eigenstates of the operator $\tau^{z}$. Here, the reduced evolution operators $U_{j}(t)$ obey the following Schrödinger equation

$$
i \hbar \partial_{t} U_{j}(t)=H_{j E}^{\prime} U_{j}(t), j=A, B,
$$

which can be computed exactly as

$$
\begin{aligned}
U_{j}\left(x_{j}, t\right) & =\exp \left[-\frac{i}{\hbar} \sum_{n=1}^{N} \int_{0}^{t} d t^{\prime} V\left(x_{j}+v_{j} t^{\prime}-x_{n}\right)\right. \\
& \left.\times\left(\sigma_{n}^{+} e^{-i \frac{\omega}{v_{j}} x_{j}}+\sigma_{n}^{-} e^{i \frac{\omega}{v_{j}} x_{j}}\right)\right]
\end{aligned}
$$

in the coordinator representation. With the SU(2) algebra, the exponential (10) can be disentangled as

$$
U_{j}\left(x_{j}, t\right)=\prod_{n} e^{-i \sigma_{n}^{+} \tan \alpha_{n}^{[j]}(t)} e^{-\sigma_{n}^{z} \ln \cos \alpha_{n}^{[j]}(t)} e^{-i \sigma_{n}^{-} \tan \alpha_{n}^{[j]}(t)}
$$

where we have defined the time-dependent tipping angles [9] of the $n$th spin as

$$
\alpha_{n}^{[j]}(t)=\int_{0}^{t} \frac{d t^{\prime}}{\hbar} V\left(x_{j}+v_{j} t^{\prime}-x_{n}\right) .
$$


For the sake of simplicity, we restrict our attention to the case of $\delta$-shaped potentials, i.e., assuming that $V(x)=V_{0} \Omega \delta(x)$. It allows us to obtain the tipping angles exactly as

$$
\alpha_{n}^{[j]}(t)=\frac{V_{0} \Omega}{\hbar v_{j}} \Theta\left(x_{j}+v_{j} t-x_{n}\right),
$$

where $\Theta(y)$ is the Heaviside unit step function, i.e., $\Theta(y)=1$ for $y>0$, and $\Theta(y)=0$ for $y<0$. In Eq. (13), we have assumed that the spin array is placed at the far right of the origin $\left(x_{1}>0\right)$, and the two particles are initially at the position $x_{A}$ and $x_{B}$ with $x_{A}, x_{B}<x_{1}$, i.e., well outside the potential region of the spin array. It can observed that if two particles, initially at the same position $x_{A}=x_{B}=x$, move with the same constant velocities, i.e., $v_{j}=v$, tipping angles of the $n$th spin are equal, $\alpha_{n}^{[j]}(t)=\alpha_{n}(t)=\frac{V_{0} \Omega}{\hbar v} \Theta\left(x+v t-x_{n}\right)$.

To show the damping effect of the $1 \mathrm{D}$ array on the spin state of the particles, we first study the time evolution of one particle (say, $A$ ) prepared initially in the state $|\psi\rangle\left|x_{A}\right\rangle$, where $|\psi\rangle=c_{0}|\downarrow\rangle+c_{1}|\uparrow\rangle$ is the superposition state of the spin-up $|\uparrow\rangle$ and spin-down $|\downarrow\rangle$ and the particle is initially located at the origin $x_{A}=0$. The initial state of the $1 \mathrm{D} N$-spin array is taken to be the ground state $\left|0_{E}\right\rangle$ (i.e. all spins down). The spin state of the particle at time $t>0$ reads

$$
\begin{aligned}
\rho^{S}(t)= & \left|c_{0}\right|^{2}|\downarrow\rangle\left\langle\left.\downarrow|+| c_{1}\right|^{2} \mid \uparrow\right\rangle\langle\uparrow| \\
& +c_{1} c_{0}^{*}|\uparrow\rangle\langle\downarrow| f(t)+\text { h.c. },
\end{aligned}
$$

where the off-diagonal elements are proportional to decoherence factor [7]

$$
\begin{aligned}
f(t) & =\left\langle 00_{E}\left|U_{A}\right| 00_{E}\right\rangle \\
& =\prod_{n} \cos \left[\frac{V_{0} \Omega}{\hbar v_{A}} \Theta\left(v_{A} t-x_{n}\right)\right]
\end{aligned}
$$

with a factorization structure. Now, we introduce the parameter

$$
q_{j}=\sin ^{2} \frac{V_{0} \Omega}{\hbar v_{j}},
$$

which is the "spin-flip" probability, i.e., the probability of dissociating one $\mathrm{AgBr}$ molecule due to the energy exchange with the $j$ th particle. Here, only one particle is considered i.e., $j=A$. For an array with $N$ spins, $\bar{n}=q N$ is the average number of dissociated molecule. In the weak-coupling macroscopic limit

$$
q_{A}=\left(\frac{V_{0} \Omega}{\hbar v_{A}}\right)^{2},
$$

and $\bar{n}=q N$ is required to be finite [9]. With the assumption that

$$
x_{n}=x_{1}+(n-1) \Delta,
$$

the decoherence factor is approximately calculated as

$$
f(t)=e^{-\frac{\bar{n}}{2}\left[\frac{v_{A} t-x_{1}}{L} \Theta\left(x_{N}-v_{A} t\right) \Theta\left(v_{A} t-x_{1}\right)+\Theta\left(v_{A} t-x_{N}\right)\right]}
$$

for $\Delta / L \rightarrow 0$ as $N \rightarrow \infty$. It can be found that the decoherence factor decays exponentially within the regime of the macroscopic medium (i.e. $x_{1}<v_{A} t<x_{N}$ ), and becomes a constant after the interaction is over. In the terminology of quantum mechanics, the qubit is subject to a phase damping channel.

\section{QUANTUM CORRELATIONS OF THE TWO QUBITS}

We regard the spins of the two particles as a two-qubit composite system and the 1D $N$-spin array as the environment. To investigate the quantum correlations of the two qubits interacting with the same environment, we assume that the two-qubit system and the environment are initially uncorrelated

$$
\rho(0)=\rho_{i n}^{S} \otimes \rho_{i n}^{D} \otimes \rho_{i n}^{E}
$$

Hereafter, the density matrixes related to the spin and spatial degrees of the particle $Q_{A}\left(Q_{B}\right)$, and the 1D $N$-spin array are discriminated by the superscripts $S, D, E$. To further distinguish the spin (spatial) degree between the particle $A$ and $B$, the superscripts $S_{\beta}\left(D_{\beta}\right)$ will be used with $\beta=A, B$. The two-qubit system is initial in a class of states with maximally mixed marginal, known as Bell-diagonal states [21]

$$
\rho_{i n}^{S}=\frac{1}{4}\left(I_{A B}+\sum_{j=1}^{3} c_{j} \tau_{j}^{A} \otimes \tau_{j}^{B}\right),
$$

which has been discussed in the literature on entanglement and its decay under decoherence [22], and quantum correlations besides entanglement $[10-15,23]$. Here, $I_{A B}$ is the $4 \times 4$ identity matrix, and $c_{j}\left(0 \leq\left|c_{j}\right| \leq 1\right)$ are real numbers satisfying the unit trace and positivity conditions of the density $\rho_{i n}^{S}$. The state in Eq. (21) becomes the Werner state when $\left|c_{1}\right|=\left|c_{2}\right|=\left|c_{3}\right|=c$ and Bell state when $\left|c_{1}\right|=\left|c_{2}\right|=\left|c_{3}\right|=1$. After the interaction the two-qubit state evolves into

$$
\rho^{S}(t)=\frac{1}{4}\left(\begin{array}{cccc}
1+c_{3} & 0 & 0 & \Lambda_{2}(t) \\
0 & 1-c_{3} & \Lambda_{1}^{*}(t) & 0 \\
0 & \Lambda_{1}(t) & 1-c_{3} & 0 \\
\Lambda_{2}^{*}(t) & 0 & 0 & 1+c_{3}
\end{array}\right)
$$

where the off-diagonal elements are time-dependent

$$
\begin{aligned}
& \Lambda_{1}(t)=\left(c_{1}+c_{2}\right) f_{1}(t), \\
& \Lambda_{2}(t)=\left(c_{1}-c_{2}\right) f_{2}(t),
\end{aligned}
$$

and the diagonal elements of the density matrix $\rho^{S}(t)$ do not change with time. In Eq. (23), the time-dependent factors are defined as

$$
\begin{aligned}
f_{1}(t)= & e^{i\left(\omega_{A}-\omega_{B}\right) t} \int d x_{A}^{\prime} d x_{B}^{\prime}\left\langle x_{A}^{\prime} x_{B}^{\prime}\left|\rho_{i n}^{D}\right| x_{A}^{\prime} x_{B}^{\prime}\right\rangle \\
& \operatorname{Tr}_{E}\left[U_{B}\left(x_{B}^{\prime}, t\right) \rho_{i n}^{E} U_{A}^{\dagger}\left(x_{A}^{\prime}, t\right)\right], \\
f_{2}(t)= & e^{-i\left(\omega_{A}+\omega_{B}\right) t} \int d x_{A}^{\prime} d x_{B}^{\prime}\left\langle x_{A}^{\prime} x_{B}^{\prime}\left|\rho_{i n}^{D}\right| x_{A}^{\prime} x_{B}^{\prime}\right\rangle \\
& \operatorname{Tr}_{E}\left[U_{A}\left(x_{A}^{\prime}, t\right) U_{B}\left(x_{B}^{\prime}, t\right) \rho_{i n}^{E}\right],
\end{aligned}
$$

where $\left|x_{j}^{\prime}\right\rangle$ is the eigenstate of the coordinator operator $\hat{x}_{j}$ of the $Q_{j}$ particle.

The QD [3, 24-27] is defined as the difference between total correlations and classical correlations in a bipartite system with the expression $\mathscr{D}\left(\rho_{A B}\right)=\mathcal{I}\left(\rho_{A B}\right)-\mathcal{J}\left(\rho_{A B}\right)$, where $\rho_{A B}$ is the density operator of the total system. Here, total correlations is equal to quantum mutual information $\mathcal{I}\left(\rho_{A B}\right)=$ 
$S\left(\rho_{A}\right)+S\left(\rho_{B}\right)-S\left(\rho_{A B}\right)$, where $S$ is the von Neumann entropy, and $\rho_{A}\left(\rho_{B}\right)$ is the reduced density matrix of the subsystem $A(B)$. Classical correlations between the two subsystems $A$ and $B$ is the largest information gained about one subsystem after a measurement of the other, and it can be captured by $\mathcal{J}\left(\rho_{A B}\right)=\max \left[S\left(\rho_{A}\right)-S\left(\rho_{A} \mid \Pi_{k}^{B}\right)\right]$, where $S\left(\rho_{A} \mid \Pi_{k}^{B}\right)$ is the entropy of $A$ after a measurement of $B$, and $\Pi_{k}^{B}$ is an orthogonal projective measurement made on $B$ with $\sum_{k} \Pi_{k}^{B}=I$ [21]. In this paper, we choose $\Pi_{k}^{B}=\left|\pi_{k}\right\rangle\left\langle\pi_{k}\right|, k=1,2$, where $\left|\pi_{1}\right\rangle=\cos \theta|0\rangle+e^{i \phi} \sin \theta|1\rangle,\left|\pi_{2}\right\rangle=e^{-i \phi} \sin \theta|0\rangle-\cos \theta|1\rangle$, with $0 \leq \theta \leq \pi / 2$ and $0 \leq \phi \leq 2 \pi$. It is worth to stress that QD is dependent on the subsystem on which the measurement is performed, and its quantity is not symmetrical in general. However, in the case of $S\left(\rho_{A}\right)=S\left(\rho_{B}\right)$, QD computed on measuring subsystem $A$ is equal to that on measuring subsystem $B$ [28]. This is an important reason why the Bell-diagonal states are chosen as the initial states of the two-qubit system in current literatures [11, 13-15].

The total correlations corresponding to the density matrix in Eq. (22) can be obtained as $\mathcal{I}\left(\rho^{S}(t)\right)=2+\sum_{i=1}^{4} \lambda_{i} \log \lambda_{i}$, where

$$
\begin{aligned}
& \lambda_{1,2}=\frac{1}{4}\left[1-c_{3} \pm\left|\left(c_{1}+c_{2}\right) f_{1}(t)\right|\right], \\
& \lambda_{3,4}=\frac{1}{4}\left[1+c_{3} \pm\left|\left(c_{1}-c_{2}\right) f_{2}(t)\right|\right]
\end{aligned}
$$

are four eigenvalues of $\rho^{S}(t)$. And the classical correlations between the two qubits is also derived as $\mathcal{J}\left(\rho^{S}(t)\right)=$ $\sum_{n=1}^{2} \frac{1+(-1)^{n} \chi}{2} \log _{2}\left[1+(-1)^{n} \chi\right]$, where

$$
\chi(t)=\max \left[\left|c_{3}\right|, \frac{\left|\left(c_{1}+c_{2}\right) f_{1}(t)\right|+\left|\left(c_{1}-c_{2}\right) f_{2}(t)\right|}{2}\right] .
$$

Therefore, QD takes the form as

$\mathscr{D}\left(\rho^{S}(t)\right)=2+\sum_{i=1}^{4} \lambda_{i} \log \lambda_{i}-\sum_{n=1}^{2} \frac{1+(-1)^{n} \chi}{2} \log _{2}\left[1+(-1)^{n} \chi\right]$.

Concurrence [29] quantifies the entanglement of the state of two-qubit system $\rho_{A B}$ and is defined as $C\left(\rho_{A B}\right)=$ $\max \left\{0, \lambda_{1}-\lambda_{2}-\lambda_{3}-\lambda_{4}\right\}$, where $\lambda_{1} \geq \lambda_{2} \geq \lambda_{3} \geq \lambda_{4}$ are square roots of the eigenvalues of the matrix $\rho_{A B}\left(\tau_{y} \otimes\right.$ $\left.\tau_{y}\right) \rho_{A B}^{*}\left(\tau_{y} \otimes \tau_{y}\right)$, with $\rho_{A B}^{*}$ denoting the complex conjugate of $\rho_{A B}$ and $\tau_{y}$ being Pauli matrix. When the density matrix of two-qubit has an $X$ structure, defined by $\rho_{12}=\rho_{13}=\rho_{24}=$ $\rho_{34}=0$, concurrence has a simple analytic expression [30]

$$
C\left(\rho_{A B}\right)=2 \max \left\{0, \Gamma_{1}, \Gamma_{2}\right\},
$$

where $\Gamma_{1}=\left|\rho_{14}\right|-\sqrt{\rho_{22} \rho_{33}}$ and $\Gamma_{2}=\left|\rho_{23}\right|-\sqrt{\rho_{11} \rho_{44}}$. For the density operator given by Eq. (22), we can get

$$
\begin{aligned}
& \Gamma_{1}=\frac{1}{4}\left[\left|\left(c_{1}-c_{2}\right) f_{2}(t)\right|-\left|1-c_{3}\right|\right], \\
& \Gamma_{2}=\frac{1}{4}\left[\left|\left(c_{1}+c_{2}\right) f_{1}^{*}(t)\right|-\left|1+c_{3}\right|\right] .
\end{aligned}
$$

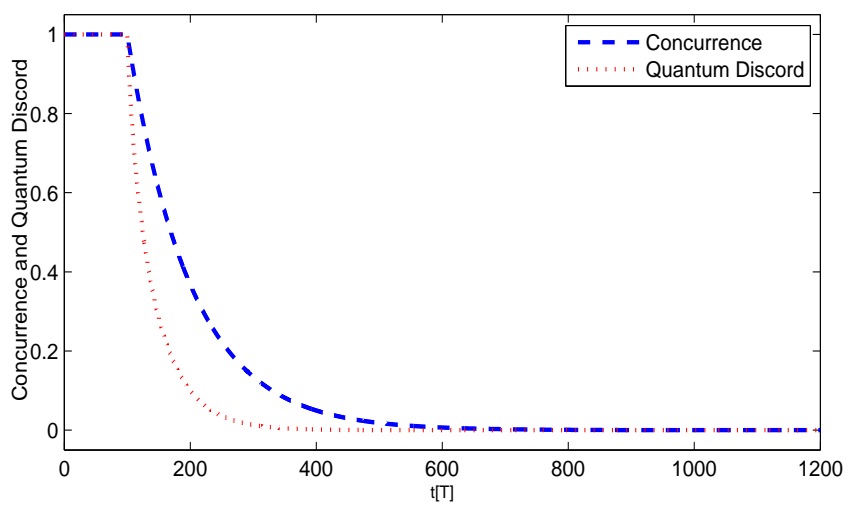

FIG. 1: (Color online). Dynamics of concurrence (blue-dashed line) and quantum discord (red-dotted line) as a function of $t$ for $c_{1}=$ $-c_{2}= \pm 1$ and $c_{3}=1$. Here, we choose $T=\Delta / v$ and $\Delta$ as a unit of time and length, respectively. Moreover, $q=0.005, N=1001, x_{1}=$ $100, x_{N}=1100, L=1000$.

\section{A. Two particles located initially at the same position}

For the sake of simplicity, we give priority to the case that the two particles are initially located at the origin $x_{A}=x_{B}=$ 0 , and have the identical velocity parameter $v_{A}=v_{B}=v$. The initial state of the macroscopic medium is taken to be the ground state $\left|0_{E}\right\rangle$. From Eq. (24), the time-dependent factor is calculated as

$$
\begin{aligned}
& f_{1}(t)=e^{i\left(\omega_{A}-\omega_{B}\right) t}, \\
& f_{2}(t)=e^{-i\left(\omega_{A}+\omega_{B}\right) t} e^{-2 \bar{n}\left[\frac{v t-x_{1}}{L} \Theta\left(x_{N}-v t\right) \Theta\left(v t-x_{1}\right)+\Theta\left(v t-x_{N}\right)\right]}
\end{aligned}
$$

in the weak-coupling macroscopic limit. We note that it is unnecessary to give the value of $\omega_{A}$ and $\omega_{B}$ because QD and concurrence only dependent on the norm of functions $f_{1}(t)$ and $f_{2}(t)$.

(1) We first consider the initial state with the following parameters: $c_{1}=-c_{2}= \pm 1, c_{3}=1$, which means the two-qubit composite system is initially in Bell states $\left|\Phi^{ \pm}\right\rangle=$ $(|00\rangle \pm|11\rangle) / \sqrt{2}$. In Fig. 11, we plot the concurrence $C\left(\rho^{S}(t)\right)=$ $\left|f_{2}(t)\right|$ and the QD $\mathscr{D}\left(\rho^{S}(t)\right)=\frac{1}{2}\left(1+\left|f_{2}(t)\right|\right) \log \left(1+\left|f_{2}(t)\right|\right)+$ $\frac{1}{2}\left(1-\left|f_{2}(t)\right|\right) \log \left(1-\left|f_{2}(t)\right|\right)$ as a function of time. It can be observed that as the time increases, the concurrence and QD are unchanged for a while, and they decrease afterward. Actually, the dynamics of the particles can be divided into three time periods. The first time period ends at the particles meeting the 1 st spin of the chain. In this time period, the state of the total system is unchanged since the particles do not interact with the macroscopic medium, therefore, the concurrence and QD keep their initial value. After the particles enter the medium, the second time period begins. Due to the energy exchange between the system and environment, the environment results in the dephasing of the two-qubit composite system, which decreases the concurrence and QD. The third time period begins after the particles have interacted with the last spin of the chain. The concurrence and QD do not change with time due to the noninteraction between the particles and the macroscopic medium. Here, both concurrence and QD decay 


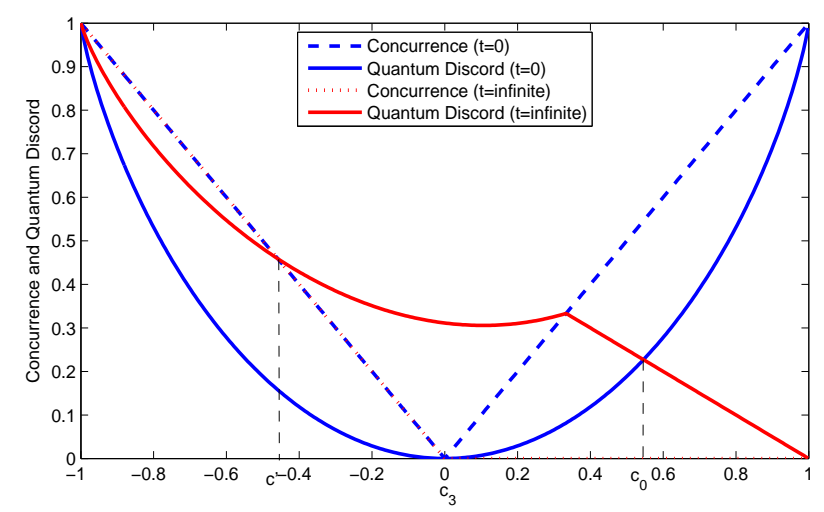

FIG. 2: (Color online). The concurrence and QD as a function of $c_{3}$ at $t=0$ and $t=\infty$ with $c_{1}= \pm 1, c_{2}=\mp c_{3}$ and $\left|c_{3}\right|<1$. The other parameters are the same as those in Fig. 1

asymptotically, and the QD decays faster than concurrence.

(2) Consider the two-qubit composite system is initially in the Bell states $\left|\Psi^{ \pm}\right\rangle=(|01\rangle \pm|10\rangle) / \sqrt{2}$, which is obtained by setting $c_{1}=c_{2}= \pm 1$ and $c_{3}=-1$ in Eq. (21). In this case, one of the off-diagonal element in Eq.(22) tends to zero, i.e., $\Lambda_{2}(t) \rightarrow 0$. The concurrence and QD keep their initial value, i.e., $C\left(\rho^{S}(t)\right)=\mathscr{D}\left(\rho^{S}(t)\right)=1$. The total correlations are equally divided into classical and quantum correlations through all the time. This phenomenon can be observed from Eq.(24), whose evolution operator $U_{A}^{\dagger}\left(x_{A}, t\right) U_{B}\left(x_{B}, t\right)$ is generated by Hamiltonian $H_{1}^{\prime}=H_{A E}^{\prime}-H_{B E}^{\prime}$. Since the inter-distance between two particles vanishes, $H_{1}^{\prime}=0$, which means the environment does not induces the loss of coherence without energy exchange. Consequently, the off-diagonal element remains unchanged in the subspace with one spin up and one spin down.

(3) Now, we consider the case with parameters $c_{1}=$ $\pm 1, c_{2}=\mp c_{3}$ and $\left|c_{3}\right|<1$ in Eq. (21), which is the mixture of Bell states mentioned above [15]

$$
\rho^{S}(0)=\frac{1+c_{3}}{2}\left|\Phi^{ \pm}\right\rangle\left\langle\Phi^{ \pm}\left|+\frac{1-c_{3}}{2}\right| \Psi^{ \pm}\right\rangle\left\langle\Psi^{ \pm}\right| .
$$

To give a preliminary change of the concurrence and QD before and after the interaction between the particles and environment, we first discuss the quantities $C\left(\rho^{S}(t)\right), \mathscr{D}\left(\rho^{S}(t)\right)$ at time $t=0$ and $t=\infty$

$$
\begin{aligned}
C\left(\rho^{S}(0)\right)= & \left|c_{3}\right| \\
C\left(\rho^{S}(\infty)\right)= & \left\{\begin{array}{c}
\left|c_{3}\right|, \text { for } c_{3}<0 \\
0, \text { for } c_{3}>0
\end{array}\right. \\
\mathscr{D}\left(\rho^{S}(0)\right)= & \frac{1}{2}\left(1-c_{3}\right) \log _{2}\left(1-c_{3}\right) \\
& +\frac{1}{2}\left(1+c_{3}\right) \log _{2}\left(1+c_{3}\right) \\
\mathscr{D}\left(\rho^{S}(\infty)\right)= & \frac{1}{2}\left(1-c_{3}\right) \log _{2}\left[2\left(1-c_{3}\right)\right] \\
& +\frac{1}{2}\left(1+c_{3}\right) \log _{2}\left(1+c_{3}\right)-\frac{1+\theta}{2} \log _{2}(1+\theta) \\
& -\frac{1-\theta}{2} \log _{2}(1-\theta)
\end{aligned}
$$

where $\theta=\max \left[\left|c_{3}\right|, \frac{1-c_{3}}{2}\right]$. Equation (32) is obtained by letting $\Lambda_{2}(t)=0$ as $\Lambda_{2}(t)$ approaches zero very fast. In Fig. 2] we plot the concurrences and QDs at $t=0$ and $t=\infty$ as a function of the parameter $c_{3}$. We note that the curve $C\left(\rho^{S}(\infty)\right)$ at $c_{3} \in(-1,0)$ overlaps the curve $C\left(\rho^{S}(0)\right)$ in Fig. 2. It can be observed that: (i) When $c_{3} \in(-1,0)$, concurrence $C\left(\rho^{S}(\infty)\right)=C\left(\rho^{S}(0)\right)$, but when $c_{3} \in(0,1), C\left(\rho^{S}(\infty)\right)=0$, which is always smaller than its initial value $C\left(\rho^{S}(0)\right)$. Actually, the variation of the concurrence before and after the interaction is due to the increasing of the probability of state $\left|\Phi^{ \pm}\right\rangle$as the parameter $c_{3}$ increases in Eq. (31). (ii) The initial QD, $\mathscr{D}\left(\rho^{S}(0)\right)$, is symmetry about the axis $c_{3}=0$. The final $\mathrm{QD}, \mathscr{D}\left(\rho^{S}(\infty)\right)$, has a sudden change at the point $c_{3}=1 / 3$, which is caused by the value of $\theta$. When $c_{3}=-1$, the state given in Eq. (31) reduces to $\left|\Psi^{ \pm}\right\rangle$, and the results presented previously is recovered. When $c_{3} \in\left(-1, c_{0}\right)$, the final QD is larger than the initial QD, i.e., $\mathscr{D}\left(\rho^{S}(\infty)\right)>\mathscr{D}\left(\rho^{S}(0)\right)$, which means QD is amplified after the two-qubit composite system interacting with the environment. As $c_{3}$ continues to increase, $\mathscr{D}\left(\rho^{S}(\infty)\right)<\mathscr{D}\left(\rho^{S}(0)\right)$. (iii) In the initial time, the QD is always smaller than concurrence, which shows that the total amount of quantum correlation is captured by an entanglement measure. However, things become different at $t=\infty$. Two curves intersect each other at $c_{3}=c^{\prime}$. When $c_{3} \in\left(-1, c^{\prime}\right)$, the QD is smaller than the concurrence. However, the QD is greater than the concurrence when $c_{3} \in\left(c^{\prime}, 1\right)$, which shows that the absence of entanglement does not necessarily indicate the absence of quantum correlation. (iv) At the point $c_{3}=0$, there is no correlation (QD and concurrence) between the two qubits since the initial state in Eq. (31) is a maximum mix state. After the interaction is completed, quantum entanglement remains unchanged, however, the QD is nonzero. Hence the interaction generates the quantum correlation.

Now, we discuss the entanglement dynamics and the time evolution of the QD. Actually, these are predicted in the above discussion. The entanglement dynamics remains unchanged over the time for $c_{3} \in(-1,0)$, which can be observed in Fig. 3 a). For $c_{3} \in(0,1)$, the concurrence first keeps its initial value when the two-qubit is away from the macroscopic medium. After the two qubits enter the macroscopic medium, the concurrence with the expression $C\left(\rho^{S}(t)\right)=$ $\frac{1}{2}\left[\left|\left(1+c_{3}\right) f_{2}(t)\right|-\left(1-c_{3}\right)\right]$ decays monotonically and becomes zero at time $t_{0}=\frac{x_{1}}{v}-\frac{L}{2 q N v} \ln \frac{1-c_{3}}{1+c_{3}}$. With $c_{3} \in(-1,1 / 3)$, the QD increases monotonically over the time after the two particles begin to interact with the macroscopic medium, and QD gradually becomes the constant $\mathscr{D}\left(\rho^{S}(\infty)\right)$ in Eq. $\left.32 \mathrm{~d}\right)$, as shown in Fig. 3 b) and the dashed line in Fig. 4 In this regime, $\chi(t)=\frac{\left|1-c_{3}\right|+\left|\left(1+c_{3}\right) f_{2}(t)\right|}{2}$, the macroscopic medium increases the quantum correlations between the two qubits. In the regime $1 / 3<c_{3}<1$, as the interaction begins, the function $\chi(t)=\frac{\left|1-c_{3}\right|+\left|\left(1+c_{3}\right) f_{2}(t)\right|}{2}$ before a specific time $t_{c}$, where $t_{c}=\frac{x_{1}}{v}-\frac{L}{2 q N v} \ln \frac{3 c_{3}-1}{1+c_{3}}$. During the period of time $t<t_{c}$, we observe an increasing of QD. At time $t>t_{c}, \chi(t)=\left|c_{3}\right|$, QD decreases gradually. Consequently, there is a sudden change in the behavior of the QD at time $t=t_{c}$, as shown in the solid and dotted line in Fig. 4. Actually, the increase of QD at time $t<t_{c}$ is due to the classical correlation decaying faster than 
(a)

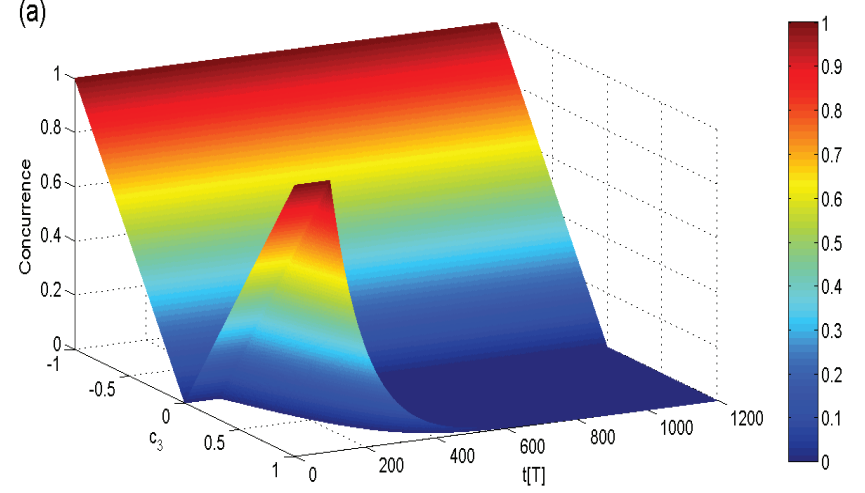

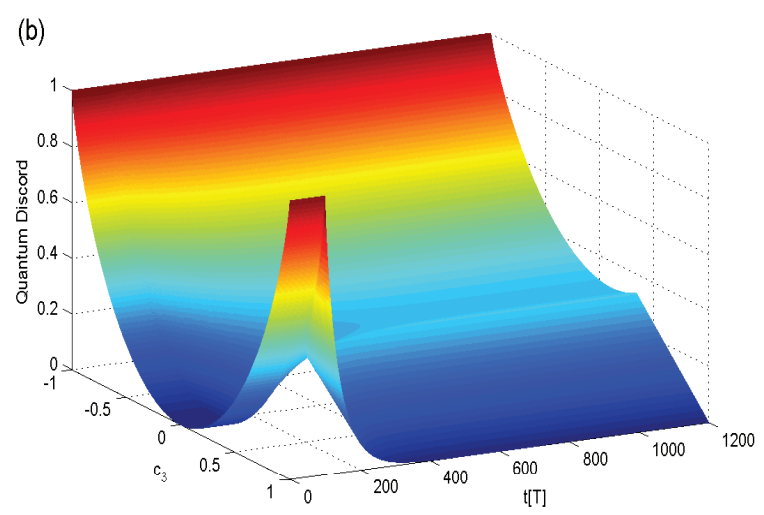

FIG. 3: (Color online). The dynamics of (a) concurrence and (b) QD with parameters $c_{1}= \pm 1, c_{2}=\mp c_{3}$ and $\left|c_{3}\right|<1$. The other parameters are the same as those in Fig. 1

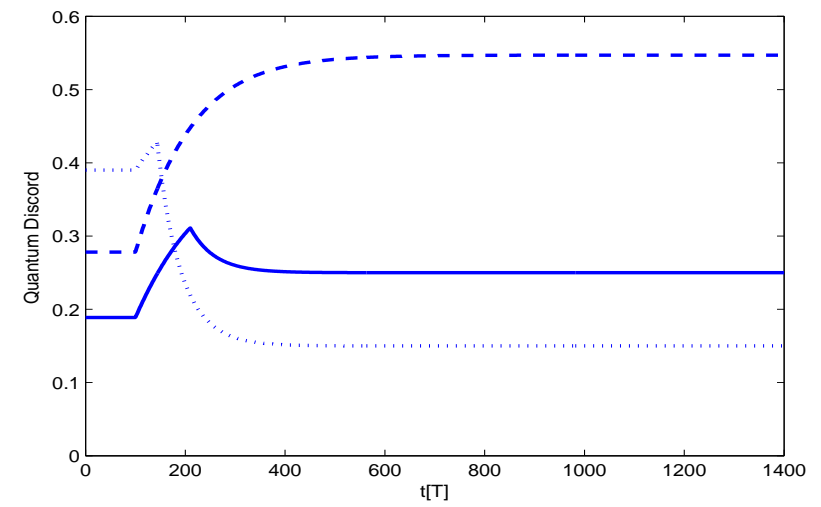

FIG. 4: (Color online). The dynamics of QD with parameters $c_{1}=$ $\pm 1, c_{2}=\mp c_{3}$ and $c_{3}=-0.6$ (dashed line), 0.5 (solid line), 0.7 (dotted line). The other parameters are the same as those in Fig. 1.

the total correlation.

\section{B. Two particles located initially at the different position}

In this section, we consider that the two particles are initially located at the different location with $x_{A}=0, x_{B}<0$, and the macroscopic medium is initially in state $\left|0_{E}\right\rangle$. The timedependent factors given in Eq. (24) read

$$
\begin{aligned}
f_{1}(t)= & e^{i\left(\omega_{A}-\omega_{B}\right) t} e^{-\frac{\bar{n}}{2}}\left[\frac{v-x_{1}}{L} \Theta\left(x_{N}-v t\right) \Theta\left(v t-x_{1}\right)+\Theta\left(v t-x_{N}\right)\right] \\
& \times e^{\frac{\bar{n}}{2}\left[\frac{x_{B}+v t-x_{1}}{L} \Theta\left(x_{N}-x_{B}-v t\right) \Theta\left(x_{B}+v t-x_{1}\right)+\Theta\left(x_{B}+v t-x_{N}\right)\right]} \\
f_{2}(t)= & e^{-i\left(\omega_{A}+\omega_{B}\right) t} e^{-\frac{\bar{n}}{2}\left[\frac{v-x_{1}}{L} \Theta\left(x_{N}-v t\right) \Theta\left(v t-x_{1}\right)+\Theta\left(v t-x_{N}\right)\right]} \\
& \times e^{-\frac{3 \bar{n}}{2}\left[\frac{x_{B}+v t-x_{1}}{L} \Theta\left(x_{N}-x_{B}-v t\right) \Theta\left(x_{B}+v t-x_{1}\right)+\Theta\left(x_{B}+v t-x_{N}\right)\right]}
\end{aligned}
$$

in the weak coupling macroscopic limit.

We plot time evolution of concurrence and QD when two qubits are initially in Bell states $\left|\Phi^{ \pm}\right\rangle$and $\left|\Psi^{ \pm}\right\rangle$in Fig. 5. It can be observed that both concurrence and quantum discord keep the initial values before two particles meeting the macroscopic medium. When particle $A$ begins to interact with the macroscopic medium, they start to decrease. After the particle $B$

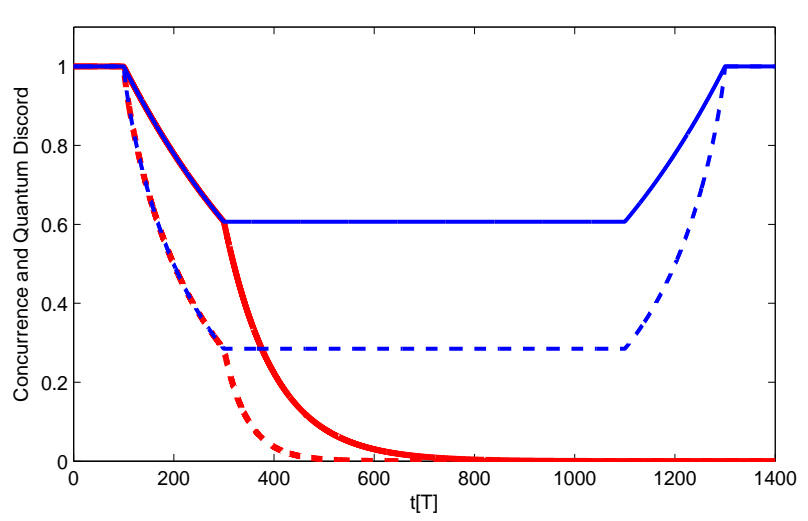

FIG. 5: (Color online). The dynamics of QD (dashed line) and concurrence (solid line) as a function of $t$ for the initial state $\Phi^{ \pm}$(red line) and $\Psi^{ \pm}$(blue line). Here, we have set $q=0.005, N=1001, x_{1}=$ $100, x_{N}=1100, x_{B}=-200, L=1000$. Time is in unit of $T=\Delta / v$, and length is in unit of $\triangle$.

enters the medium, both particles interact with the medium. However, the concurrence and QD have different behavior for different initial states: For initial state $\left|\Phi^{ \pm}\right\rangle$, both concurrence and QD decay faster than before, and vanish later; For initial state $\left|\Psi^{ \pm}\right\rangle$, the concurrence and QD remain a constant when both particles interact with the medium, and they increase after the particle $A$ left the medium and finally reach their initial value at the time that the interaction ends. Such process can be regarded as particle $\mathrm{B}$ erasing the which-path information encoded in the medium. As the average number of dissociated molecule $\bar{n}$ becomes larger enough, both concurrence and QD decay to zero asymptotically as the interaction begin, however, they were revived after a period of time for two qubits initial in state $\left|\Psi^{ \pm}\right\rangle$.

Now, we assume that the two-qubit system is initial in the mixture state given in Eq.(31). Figure 2 also gives the behavior of concurrence and QD before and after two particles interacting with the medium. Consequently, the amplification and generation of QD can also be observed in this case. To show the difference between the previous case and the one considered here, we plot the time evolution of concurrence and QD 

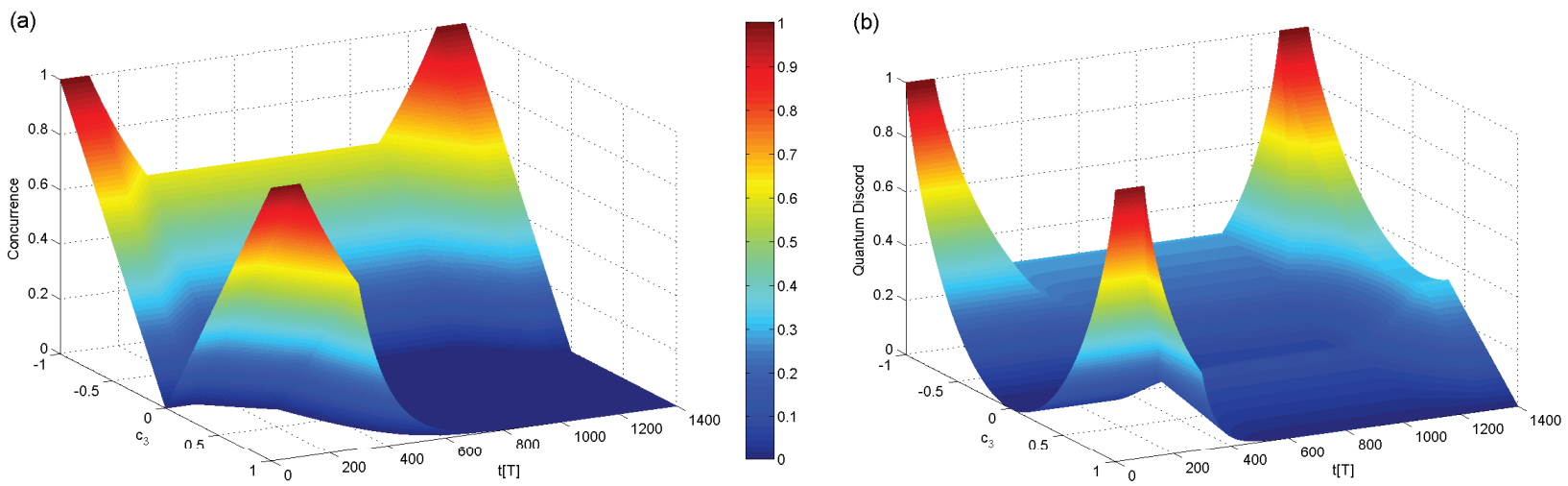

FIG. 6: (Color online). The dynamics of (a) concurrence and (b) QD with parameters $c_{1}= \pm 1, c_{2}=\mp c_{3}$ and $\left|c_{3}\right|<1$. The other parameters are the same as those in Fig. 5

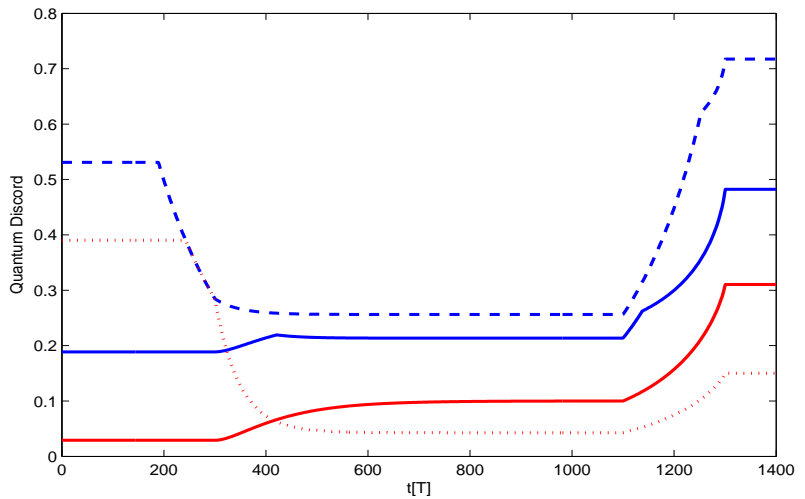

FIG. 7: (Color online). The dynamics of QD with parameters $c_{1}=$ $\pm 1, c_{2}=\mp c_{3}$ and $c_{3}=-0.8$ (blue-dashed line), -0.5 (blue-solid line), 0.2 (red-solid line), 0.7 (red-dotted line). The other parameters are the same as those in Fig.5

as the functions of parameter $c_{3}$ and time in Figs. 6a) and 6(b), respectively. It can be observed from Fig. 6a) that after the interaction begins, entanglement first decreases, then remains unchanged for a while, finally increases to its initial value in the regime $c_{3} \in(-1,0)$. Actually, one can observed first a sudden death and latter a sudden birth as the average number of dissociated molecule $\bar{n}$ becomes larger enough when the probability of state $\left|\Psi^{ \pm}\right\rangle$is larger than that of state $\left|\Phi^{ \pm}\right\rangle$in Eq. (31). In the regime $c_{3} \in(0,1)$, only the sudden death appears. Figs. 6 (b) depicts that QD experiences a decline after the interaction begins, then remains unchanged for a while, finally revives in the case of smaller $c_{3}$. However, for larger $c_{3}$, there is no obvious revival. Figs. 7 gives the QD as a function of time for a given parameter $c_{3}$. Here, it is can be find that the dynamic of QD is divided into five time periods. The first time period ends at particle $A$ meeting the 1 st spin of the chain. In this time period, the state of two-qubit is unchanged, then QD keeps its initial value $\mathscr{D}\left(\rho^{S}(0)\right)$. After particle $A$ enters the medium, the second time period begins, and it ends at particle $B$ meeting the 1st spin of chain. During this period, since $f_{1}(t)=f_{2}(t)$, QD first keeps unchanged until the critical time $\bar{t}=\frac{x_{1}}{v}-\frac{2 L \ln \left|c_{3}\right|}{q^{N v}}$, then decays monotonously, which experiences a sudden change [15]. However, such sudden change may disappears as long as $\left|c_{3}\right| \leq e^{\frac{q N X_{B}}{2 L}}$ as shown in the solid lines of Fig. 7. The third time period is the period of both two particles interacting with the macroscopic medium. The QD may either increases (see the red solid line in Fig. 7) or decreases (see the blue dashed line and red dotted line in Fig. (7), and even has a sudden change (see the blue solid line in Fig. 7), depending on the value of $c_{3}$. The fourth time period begins with particle $A$ leaving the medium and particle $B$ still interacting with the macroscopic medium. In the case that $\chi(t)=\frac{\left|\left(1-c_{3}\right) f_{1}(t)\right|+\left|\left(1+c_{3}\right) f_{2}(t)\right|}{2}$ for a given $c_{3}$, the QD increases continuously and monotonously to the value of $\mathscr{D}\left(\rho^{S}(\infty)\right)$ as shown in the red-solid line in Fig. 7 And in the case that $\chi(t)=\left|c_{3}\right|$, the QD also has a continuous and monotonous increase as shown in the red-dotted line in Fig. 7 However, in the case that $\chi(t)$ is a piecewise function of time since there exists a cross between $\left|c_{3}\right|$ and $\frac{\left|\left(1-c_{3}\right) f_{1}(t)\right|+\left|\left(1+c_{3}\right) f_{2}(t)\right|}{2}$, the QD increases fast firstly and slow later as shown in the blue-dash line and blue-solid line in Fig. 7 The last time period begins after particle $B$ have interacted with the last spin of the chain. the QD does not change with time due to the noninteraction between the particles and the macroscopic medium.

\section{DISCUSSION AND CONCLUSION}

We have investigated the dynamics of concurrence and QD with an exactly solvable model where qubits interact with a common zero-temperature reservoir. The behaviors of both entanglement and QD are presented in the initial states and the same reservoir condition. It is found that regardless the inter-distance between the two particles, the final entanglement can neither be generated nor become larger than its initial value via the interaction. However, QD can be amplified, and even be generated. Whether the inter-distance of the two particles is absence or not has significant influence on the dynamics of both entanglement and QD: 1) With a vanishing inter-distance, entanglement either remains unchanged or decay monotonically through all the time, instead, QD could either keep unchanged or increase monotonically, and it could 
even increase first and decrease later, i.e., it undergoes a sudden change. 2) With a nonzero inter-distance, both entanglement and QD varied with time. when the average number of dissociated molecule $\bar{n}$ become larger enough, entanglement experiences first a sudden death and later a sudden birth in the time evolution, but QD begins to decay to zero asymptotically and has a rival later.

\section{Acknowledgments}

This work was supported by NSFC Grants No. 11374095, No. 11422540 , No. 11434011 and No. 11105050; NBRPC
Grants No. 2012CB922103; Hunan Provincial Natural Science Foundation of China Grants No. 11JJ7001 and No. 12JJ1002; and Scientific Research Fund of Hunan Provincial Education Department Grant No. 11B076.
[1] E. Knill and R. Laflamme, Phys. Rev. Lett. 81, 5672 (1998).

[2] K. Modi, A. Brodutch, H. Cable, T. Paterek, and V. Vedral, Rev. Mod. Phys. 84, 1655 (2012)

[3] H. Ollivier and W. H. Zurek, Phys. Rev. Lett. 88, 017901 (2001); W. H. Zurek, Phys. Rev. A 67, 012320 (2003).

[4] L. Henderson and V. Vedral, J. Phys. A 34, 6899 (2001).

[5] K. Hepp, Helv. Phys. Acta 45, 237 (1972).

[6] H. Nakazato and S. Pascazio, Phys. Rev. Lett. 70, 1 (1993); Phys. Rev. A 48, 1066 (1993).

[7] C.P. Sun, Phys. Rev. A 48, 898 (1993).

[8] K. Hiyama and S. Takagi, Phys. Rev. A 48, 2586 (1993).

[9] H. Nakazato, M. Namiki, and S. Pascazio, Phys. Rev. Lett. 73, 1063 (1994).

[10] T. Werlang, S. Souza, F.F. Fanchini, and C.J. Villas Boas, Phys. Rev. A 80, 024103 (2009).

[11] J. Maziero, L.C. Céleri, R. M. Serra, and V. Vedral, Phys. Rev. A 80, 044102 (2009).

[12] B. Wang, Z.Y. Xu, Z.Q. Chen, and M. Feng, Phys. Rev. A 81, 014101 (2010).

[13] J. Maziero, T. Werlang, F. F. Fanchini, L.C. Céleri, and R. M. Serra, Phys. Rev. A 81, 022116 (2010).

[14] F.F. Fanchini, T. Werlang, C.A. Brasil, L.G.E. Arruda, and A.O. Caldeira, Phys. Rev. A 81, 052107 (2010).

[15] L. Mazzola, J. Piilo, and S. Maniscalco, Phys. Rev. Lett. 104, 200401 (2010).
[16] F. Altintas and R. Eryigit, Phys. Rev. A 87, 022124 (2013).

[17] N. Doustimotlagh, S. Wang, C. You, G. Long, Quantum. inf. Comput. 7, 459 (2007).

[18] P. Zhang, B. You, L. Cen, Chin. Sci. Bull. 59: 3841-3846 (2014).

[19] C. Yao, Z. Chen, Z. Ma etc, Quantum. inf. Comput. 7, 459(2007).

[20] J. Guo, H. Li, G. Long, Quantum. inf. Comput. 7, 459 (2007).

[21] S. Luo, Phys. Rev. A 77, 042303 (2008).

[22] T. Yu and J. H. Eberly, Phys. Rev. Lett. 93, 140404 (2004); Science 323, 598 (2009).

[23] M. Ali, A.R.P. Rau, and G. Alber, Phys. Rev. A 81, 042105 (2010).

[24] J. Zhang and A. Chen, Quant. Phys. Lett. Vol. 1, No. 2, 6977(2012).

[25] X. Ma, Y. Qiao, G. Zhao etc, SCI CHINA-Phys Mech Astron, 56(3):600-605 (2013).

[26] X. Su, Chin. Sci. Bull. 59: 1083-1090 (2014).

[27] T. Zhou, J. Cui, G. Long, Physical Review A 84 (6), 062105 (2011).

[28] V. Vedral, Phys. Rev. L 90, 050401 (2003).

[29] W.K. Wootters, Phys. Rev. L 80, 2245 (1998).

[30] T. Yu, J.H.Eberly, Quantum. inf. Comput. 7, 459 (2007). 